\title{
On the Reciprocity of TEM and STEM
}

\author{
H. Rose and C. Kisielowski
}

Lawrence Berkeley National Laboratory, One Cyclotron Rd. Berkeley CA. 94720

Reciprocity is a wave optical argument first raised by Helmholz in 1860 for optical instruments stating that ray trajectories can be inverted because of the nature of the stationary wave equation. This equation is of second in the derivative with respect to the $\mathrm{z}$-coordinate. Since this coordinate is generally chosen along the optic axis, image and source points can be exchanged. In particular, the source plane in the CTEM corresponds to the detector plane in the STEM and this equivalence was pointed out in the past on numerous occasions ${ }^{1,2,3,4}$. The reciprocity theorem applies for the wave function of elastically scattered electrons and not for inelastically scattered electrons or intensities. However, we can include inelastic scattering by exchanging ground and excited states in the sample together with the source and detector points. An exchange of source and detector implies that the illumination system in the STEM must be coherent in order to obtain a diffraction-limited spot on the sample, while in the CTEM the optical system in the region between the object and the image plane must provide coherent imaging. This difference is the reason why inelastically scattered electrons affect the resolution in CTEM but not in the STEM. The geometry of the detector determines the degree of coherence of STEM image formation whereas the illumination geometry defines the coherence in the CTEM. Accordingly, the detector angle $\square$ corresponds to the illumination angle in CTEM in the case of an extended incoherent source, as shown in figure 1 . In this figure, the angle $\square$ defines the incoherent illumination angle in CTEM and the detector angle in STEM, respectively. The objective aperture angles $\square_{1}=\square_{0}(\square-1)^{1 / 2}$ and $\square_{2}=\square_{0}(\square+1)^{1 / 2}$ are determined by the angle $\square_{0}=(\square / \mathrm{Cs})^{1 / 4}$ and the normalized defocus $\square=\square \mathrm{f}(\mathrm{Cs} \square)^{-1 / 2}$. Here $\square$ is the wavelength, $\square \mathrm{f}$ the defocus, and Cs the spherical-aberration coefficient of the objective lens.

Phase contrast with parallel illumination in the CTEM corresponds to a STEM image recorded with a point detector on the optical axes. Although both images are equivalent, the doses differ considerably for identical signal/noise ratios because a point-like detector collects only a very small fraction of all scattered electrons. We can significantly increase the collection efficiency for brightfield STEM by employing segmented bright-field detectors that enable image formation by difference signals. We have shown this procedure schematically in figure 2. Annular dark-field STEM imaging is equivalent to hollow-cone TEM imaging. Resolution improvement in DF TEM for parallel illumination is feasible (Figure 3) by inserting an annular aperture in the back focal plane of the objective lens instead of a central beam stop (Figure 1) ${ }^{5}$. However, the ADF STEM detector collects scattered electrons more efficiently than the objective aperture in TEM. With increasing resolution the TEM collection efficiency increases while that of the STEM decreases because an increasing fraction of the scattered electrons remain within the illumination cone. In order to determine the atomic structure of solids, we must know the amplitude and phase of the exit wave. One can be tackle this task easier for the CTEM using parallel illumination than for the STEM because the conical illumination results in a coherent superposition of many exit waves each attributed to a plane incident wave with a different angle of incidence. We face the same convolution difficulty in STEM holography ${ }^{6,7}$.

[1] J. M. Cowley, Appl. Phys. Lett. 15, 1969, 58

[2] H. Rose, Optik 39, 1974, 416 
[3] H. Rose, Ultramicroscopy 2, 1977, 251

[4] J.C.H. Spence, J.M. Zuo, in "Electron Diffraction" Plenum, New York. 1992, p 177 ff.

[5] S. Bals, B. Kabius, M. Haider, V. Radmilovic, C. Kisielowski, Solid State Communications 130, 2004,675

[6] The authors acknowledge fruitful discussions with J.C.H. Spence on reciprocity.

[7] This work was supported by the Director, Office of Science, Office of Basic Energy Sciences, of the US Department of Energy under Contract No. DE-AC03-76SF00098.

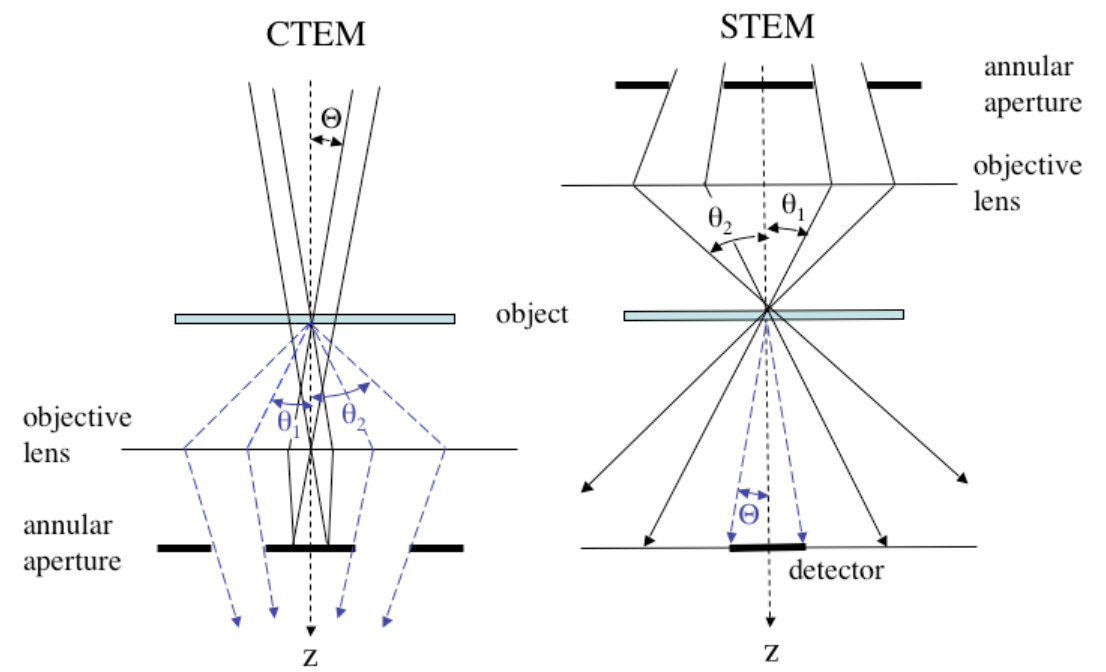

CTEM
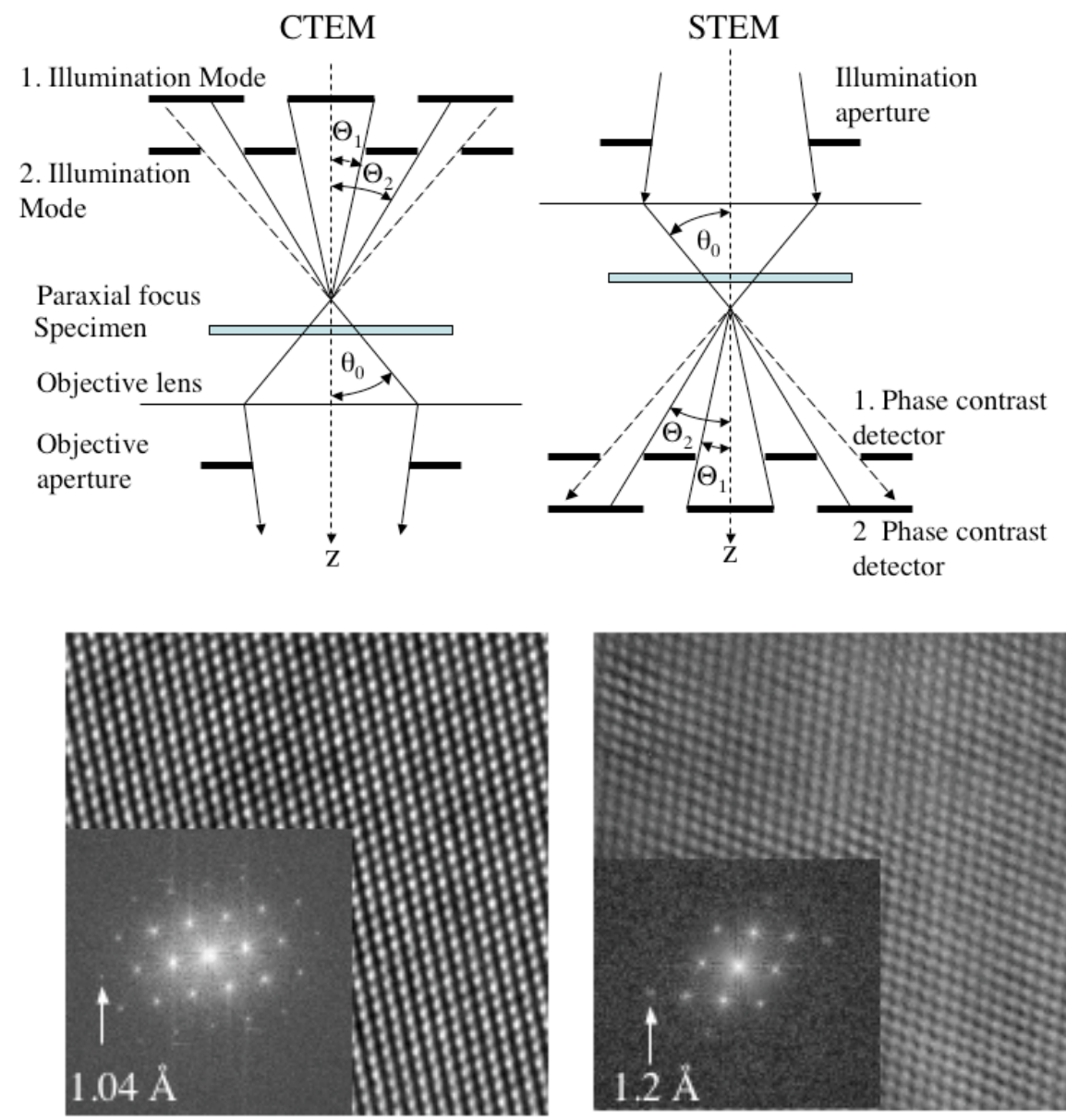

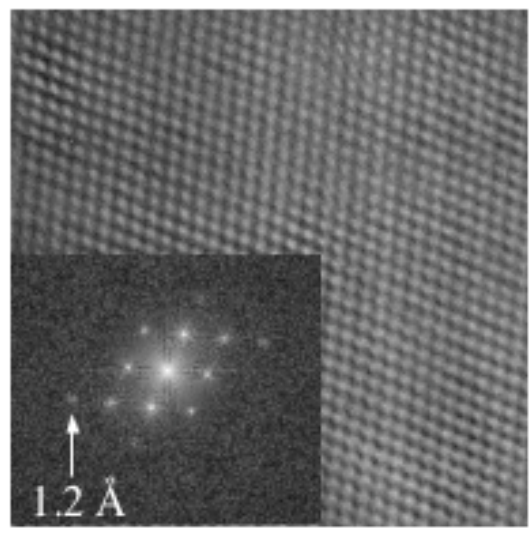

Figure 1: Ray diagram and arrangement of detectors and apertures in the CTEM and the STEM operating at equivalent dark-field conditions

Figure 2: Ray diagram and arrangement of detectors and apertures in the CTEM and the STEM operating at equivalent bright-field conditions

Figure 3: (Left) Lattice image of $\mathrm{Si}$ [110] at Scherzer defocus. (Right): Annular darkfield TEM image of the same sample area obtained by means of an annular dark-field aperture. 